\title{
Mobile Phone Assisted 3D Extra Oral Scanner for Acquiring Dental Digital Models - An Innovative Approach
}

\author{
Kapoor Abhijeet ${ }^{1}$, Eesha Mody², J. Brintha Jei ${ }^{3}$, Peter John4, Murugesan Krishnan5, B. Muthukumar ${ }^{6}$ \\ 1, 2, 3, 4, 5, 6 Department of Prosthodontics, SRM Dental College, Chennai, Tamil Nadu, India.
}

An accurate impression of the edentulous arch is of utmost importance in the rehabilitation of completely edentulous cases. The steps that follow the impression making are highly influenced by the accuracy of the impression.

In order to overcome the manual inadequacies and to improve the accuracy, various advancements have been made manually as well as digitally. Computer-aided design/computer-aided manufacturing (CAD / CAM) is one such digital advancement, which primarily aims at scanning the edentulous arches, followed by subsequent designing of the dentures and processing through various methods such as milling or 3d printing. A major drawback of using CAD / CAM technology is its high cost.

Photogrammetry, as a proof of concept, works on the principle of obtaining reliable information about physical objects through the process of recording, measuring and interpreting photographic images. Recently photogrammetry has been gaining interest in the field of dentistry. It can be used as a more cost-effective alternative for diagnosis, treatment planning and fabrication of surgical guides and in maxillofacial prosthetics.

In the current article, a novel 360-degree mobile phone scanner was devised which rotates around the object and takes images in order to compute a $3 \mathrm{~d}$ image, which can be further used for treatment planning and fabrication of dentures.

\author{
Corresponding Author: \\ Dr. Kapoor Abhijeet, \\ Department of Prosthodontics, SRM Dental \\ College, Chennai, Tamil Nadu, India. \\ E-mail: Kapoorabhijeet9@gmail.com \\ DOI: $10.14260 / \mathrm{jemds} / 2021 / 375$ \\ How to Cite This Article: \\ Abhijeet K, Mody E, Jei JB, et al. Mobile \\ phone assisted $3 D$ extra oral scanner for \\ acquiring dental digital models - an \\ innovative approach. J Evolution Med Dent \\ Sci 2021;10(23):1815-1818, DOI: \\ $10.14260 /$ jemds/2021/375
}

Submission 31-01-2021,

Peer Review 06-04-2021,

Acceptance 14-04-2021,

Published 07-06-2021.

Copyright (C) 2021 Kapoor Abhijeet et al. This is an open access article distributed under Creative Commons Attribution License [Attribution 4.0 International (CC BY 4.0)] 


\section{BACKGROUND}

The third industrial revolution, with the aid of computers and the internet, revolutionised the development process. ${ }^{1}$ As a consequence, we have now entered the fourth industrial revolution that focuses on cracking the barrier that discriminates between physical and digital worlds, enabling cyber-physical structures to be developed. ${ }^{2}$ In all disciplines, the uses of these methods can be seen and have now steadily crept their way into dentistry. The use of this technology in dentistry is primarily intended for the diagnosis, preparation and implementation of various treatments. ${ }^{3}$ The dentist will bring greater predictability and less chair time to their procedures with the introduction of these methods into clinical practice. Converting a real-time object into a digital model is a common feature of these technologies; there are some substitutes for this method. While the use of computed tomography for bone reconstruction is suggested, there are more effective ways to reproduce dental and oral structures while exposing the recipient to radiation, like 3D scanning.4

With the use of dental scanners, it is possible to obtain digital records with high precision and accuracy, being similar or superior to traditional impressions in some cases. ${ }^{4}$ The high cost of the devices, however, can still be an obstacle factor for dentists, limiting the digital implementation in their clinics. For this reason, developing low-cost techniques such as photogrammetry is interesting. It is a mathematical method of defining the spatial structure of an object based on the generation of three-dimensional coordinates by locating repeated points in multiple images obtained from different angles of the same object. ${ }^{5}$

In scientific studies, such as assessing the growth of biological samples in crop media, developing plant structures and obtaining medical parameters from patients, this technique has been used with great reliability.6-8 Photogrammetry has also been used in the field of dentistry to acquire digital models by taking intraoral and extra-oral images, and is also a very useful method for preparing and assessing the outcomes of maxillofacial surgery by providing a good soft tissue register. ${ }^{9}$ The digital camera was used to capture and record the images in this process. This article describes the use of a mobile phone camera to obtain 2D object images, and these images have been turned into a 3D digital model using free software, making it even more cost-effective.

\section{METHODS}

1. Design the prototype using (Autodesk fusion 360, California, USA). It consisted of two circular platforms which was 10 inches in diameter, a rotating device and a mobile phone holder.

2. The two-part circular platform was prepared and the pieces were printed in four segments for ease of printing and were later connected with the aid of connectors. Each of the circular platform segments measured around 11 / 2 inches in width.

3. The sections of the designed circular platform were printed using a polylactic acid material (Rever Industries, India) Fusion Deposition Modelling 3D printer (Creality Ender3, China) (Figure 1).

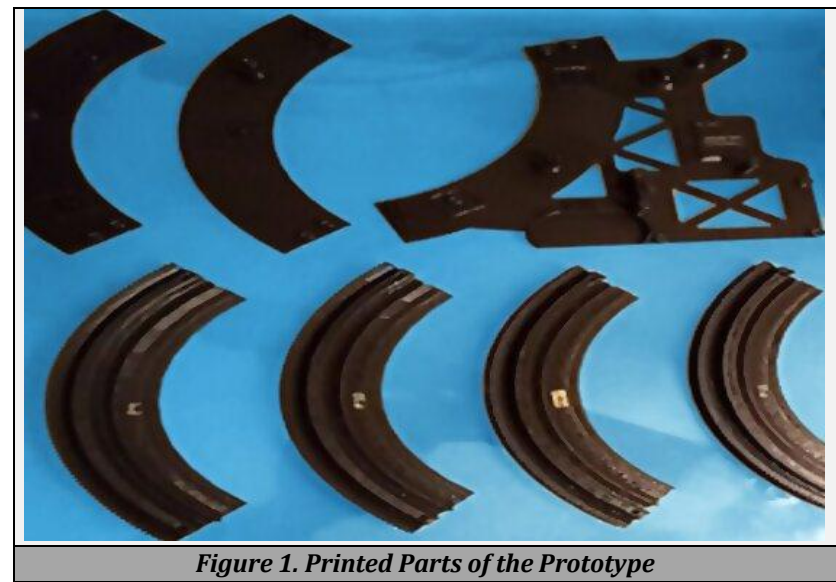

4. The rotary unit (Tower Pro Micro Servo 9 g, Italy) was attached to the circular platform and connected to it by a mobile phone holder (Bosynoy Vogue, India).

5. The Arduino board (Arduino uno SMD R3, Italy) was used to calibrate this rotating mechanism to stop the platform after $8^{0}$ rotation (Figure 2 ).

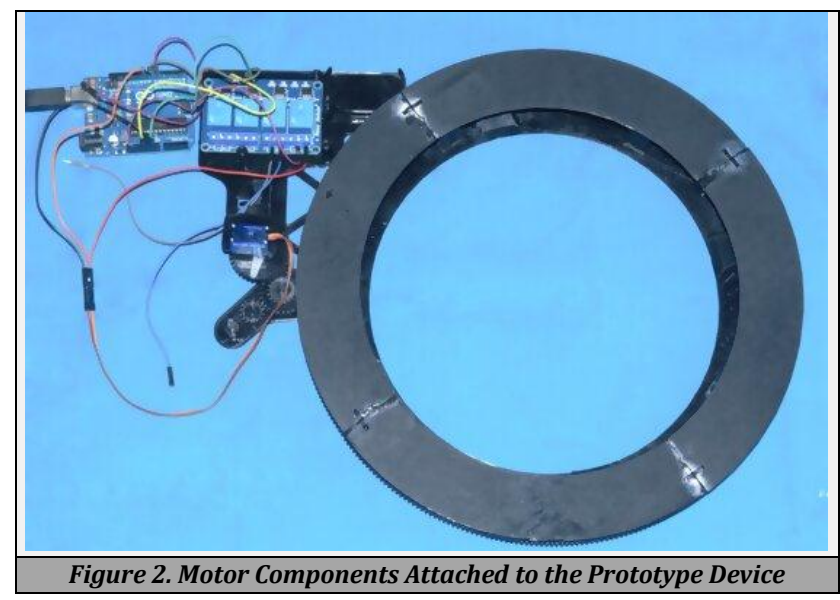

6. Maxillary edentulous cast was made using a preliminary impression of an edentulous mould with irreversible hydrocolloid impression material (Zelgan plus; Dentsply Sirona).

7. Using a straight fissure carbide bur (Dentmark, India), four holes on the ridge of the cast were drilled. In the canine region, called $\mathrm{C} 1$ and $\mathrm{C} 2$, two holes were made and the other two holes were made in the molar region, M1 and $\mathrm{M} 2$ respectively (Figure 3 ).

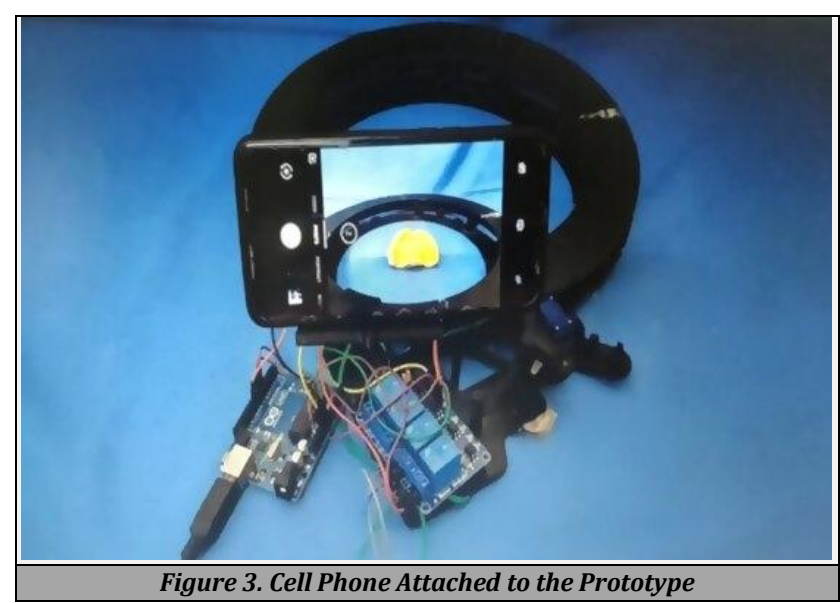


8. An elastomeric impression (DPI Photosyl, Mumbai, India) was made using putty and light body so that the holes made on the cast were recorded as elevations on the impressions (Figure 4).

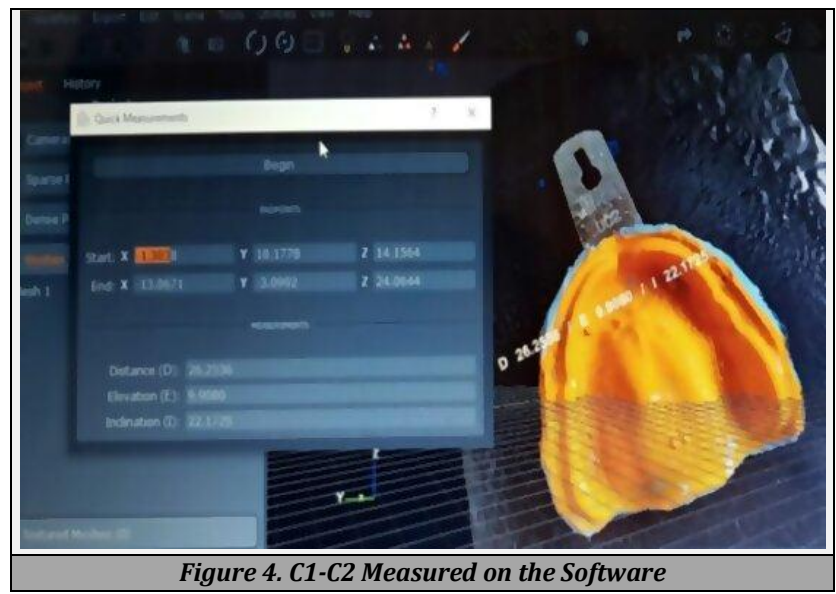

9. Soon after the impression was recovered from the cast, measurements were made at four points called C1-C2, M1-M2, C1-M1, C2-M2 using a digital Vernier caliper. (Sylvac 1 Micron Digital caliper, Mumbai).

10. The impression was mounted using a wooden framework in the centre of the circular platform and the cell phone was positioned at a radius of 7 inches to achieve a dense point cloud.

11. The pre-calibrated Arduino motor was used to rotate the revolving unit and take pictures of the impression using a 20 MP rear camera mobile phone (One plus $6 \mathrm{~T}$ mobile phone, China).

12. One image was clicked with a bluetooth remote control (OSVETA, India) when the spinning system stops every 8 degrees.

13. A total of 45 images were taken and transferred to 3DF Zephyr software (3D Flow, Italy) in order to obtain one 3D model.

14. This programme combines the acquired images and generates a cloud of scattered points in two or more images and recognises the 3D location of common points. It creates a dense cloud of dots and helps to create a 3D image of the object.

15. The distance was measure between the 3D model points C1- C2, M1-M2, C1-M1 and C2-M2 using the software measuring tool.

16. Measurements obtained from the 3D model were accurate when compared with the digital vernier caliper values.

\section{DISCUSSION}

The main objective of this study is to examine the use of photogrammetry as a viable method for the patient impression to generate digital images. That can be proven to be a powerful tool for the dentist who cannot afford the costly equipment to have a better prosthesis. Geometric and thematic information could be provided by photogrammetry. Geometric data was based on measurements of picture coordination and was represented as files of data. By automated image analysis, the thematic information is generated.10 This is a low-cost substitute for obtaining digital models compared to dental scanners.

Typically, a patient's 3D model can be obtained from cone beam computed tomography (CBCT) technology and CAD / CAM scanning, but these expensive methods cannot be used in day-to-day practice and there are also some drawbacks of CBCT in the reconstruction of 3D oral structures. ${ }^{11}$ Another downside to using CBCT is that the oral cavity artefacts can have a significant effect on the accuracy of the image generated, so the images created by CBCT might not be that accurate. When making CBCT, the patient is often exposed to radiation, which is not justified just for dental scanning. ${ }^{12}$ To remove the CBCT radiation exposure factor for patients, the authors recommended exposing the impressions to CBCT in order to acquire digital models. But the issue posed by this approach was that soon after making them, the dentist should scan the impressions, as there might be a distortion if the impression is left for a longer time. The dentist should have a fundamental understanding of the CBCT scanner, and the software used. ${ }^{13}$ The initial need for high investment and proximity to service centres is also a constraint for digital scanning technology.

The plaster models were scanned earlier to obtain a digital model. But technological development has led to the invention of intraoral scanners. In contrast to the models obtained by scanning the patient's plaster models, they were more accurate. ${ }^{14}$ Photogrammetry was performed using special equipment for imaging and processing. Recently, because of the advancement of photography and high-end cameras, this approach can be used to produce better and more precise results at comparatively lower costs. Ravasini et al. identified the great versatility of photogrammetry with good accuracy and minimal cost. ${ }^{15}$ According to Bratos et al. photogrammetry of full arch recording is a viable method when compared to conventional implant impressions. ${ }^{16}$ Whereas, using photogrammetry 3D images, Rivara et al. analysed the difference between the clinical location and the digitally planned location of dental implants and found that it was a viable and reproducible technique. ${ }^{17}$ Stuani et al. suggested that photogrammetry was a good method for diagnosis, treatment planning and management of documentation. ${ }^{18}$ While the authors conducted photogrammetry using a digital camera in previous studies, a mobile phone camera was used instead of a digital camera in this research. For this technique, free software was used that was freely accessible online and could easily be downloaded, minimising the initial investment needed for the technique. The findings from this study showed that there was some slight difference between the physical impression and the digital model about 45 microns. This may be due to the use of a low-resolution mobile phone camera, and the use of a higher resolution camera will minimise this.

\section{CONCLUSIONS}

Photogrammetry is one of the methods of 3D scanning and is useful in different areas of the medical field and can also be used in dental applications. In this paper, we have illustrated the use of photogrammetry in treatment planning and patient 
education as well. A mobile phone camera was used to make this method cost-effective in order to reduce the cost of the digital camera. This approach has been shown to be accurate to 0.5 microns and the small differences can be minimised by using a mobile phone camera that can capture high-resolution images and can further improvise the software used to achieve better performance. This technique of making digital models can also be promoted clinically in the future.

Financial or other competing interests: None.

Disclosure forms provided by the authors are available with the full text of this article at jemds.com.

\section{REFERENCES}

[1] Greenwood J. The Third Industrial Revolution: technology, productivity and income inequality. American Enterprise Institute for Public Policy Research 1997.

[2] Schwab K. The Fourth Industrial Revolution. New York: Crown Business 2017.

[3] Hultin M, Svensson KG, Trulsson M. Clinical advantages of computer-guided implant placement: a systematic review. Clin Oral Implants Res 2012;23(Suppl 6):124-35.

[4] Nedelcu R, Olsson P, Nystrom I, et al. Accuracy and precision of 3 intraoral scanners and accuracy of conventional impressions: a novel in vivo analysis method. J Dent 2018;69:110-8.

[5] Kraus K. Photogrammetry: geometry from images and laser scans. Berlin: De Gruyter Company, 1998.

[6] Syngelaki M, Hardner M, Oberthuer P, et al. A new method for non-invasive biomass determination based on stereo photogrammetry. Bioprocess Biosyst Eng 2018;41(3):369-80.

[7] Biskup B, Scharr H, Schurr U, et al. A stereo imaging system for measuring structural parameters of plant canopies. Plant Cell Environ 2007;30(10):1299-308.
[8] Mitchell HL, Newton I. Medical photogrammetric measurement: overview and prospects. ISPRS J Photogramm Remote Sens 2002;56(5-6):286-94.

[9] Kulczynski FZ, De Oliveira Andriola F, Deon PH, et al. Postural assessment in class III patients before and after orthognathic surgery. Oral Maxillofac Surg 2018;22(2):143-50.

[10] Torlegfird K. Sensors for photogrammetric mapping: review and prospects. ISPRS Journal of Photogrammetry Remote Sensing 1992;47(4):241-62.

[11] Tsapaki V. Radiation protection in dental radiologyrecent advances and future directions. Phys Med 2017;44:222-6.

[12] Ferreira JB, Christovam IO, Alencar DS, et al. Accuracy and reproducibility of dental measurements on tomographic digital models: a systematic review and meta-analysis. Dentomaxillofac Radiol 2017;46(7):20160455.

[13] Lee SM, Hou Y, Cho JH, et al. Dimensional accuracy of digital dental models from cone-beam computed tomography scans of alginate impressions according to time elapsed after the impressions. Am J Orthod Dentofacial Orthop 2016;149(2):287-94.

[14] Miyazaki T, Hotta Y, Kunii J, et al. A review of dental CAD/CAM: current status and future perspectives from 20 years of experience. Dent Mater J 2009;28(1):44-56.

[15] Ravasini F, Fornari M, Bonanini M. Quantification of the amount of dental material removed by selective grinding in wax dentures with photogrammetric measurements. Minerva Stomatol 2016;65(6):335-42.

[16] Bratos M, Bergin JM, Rubenstein JE, et al. Effect of simulated intraoral variables on the accuracy of a photogrammetric imaging technique for complete-arch implant prostheses. J Prosthet Dent 2018;120(2):232-41.

[17] Rivara F, Lumetti S, Calciolari E, et al. Photogrammetric method to measure the discrepancy between clinical and software-designed positions of implants. J Prosthet Dent 2016;115(6):703-11.

[18] Stuani VT, Ferreira R, Manfredi GGP, et al. Photogrammetry as an alternative for acquiring digital dental models: a proof of concept. Med Hypotheses 2019;128:43-9. 